DOI: $10.2478 /$ awutm-2014-0008 DE GRUYTER
Analele Universităţii de Vest,

Timişoara

Seria Matematică - Informatică

LII, 1, (2014), 121- 140

\title{
On the Solutions of a Class of Nonlinear Integral Equations in the Banach Algebra of the Continuous Functions and Some Examples
}

İsmet Özdemir, Bekir İlhan, and Ümit Çakan

\begin{abstract}
In this paper, we study the existence of the solutions of a class of functional integral equations which contain a lot of classical nonlinear integral equations as special cases. We consider the solvability of the equations in the Banach algebra of continuous functions on a closed and bounded interval. The main tools here are the measure of noncompactness and the suitable fixed point theorem for the product of two operators in the Banach algebra.
\end{abstract}

AMS Subject Classification (2010). Primary: 45M99, Secondary: 47H09.

Keywords. Nonlinear integral equation, Measure of noncompactness, Fixed point theorem, Banach algebra, Product of two operators.

\section{Introduction}

The aim of this paper is to consider the existence of the solutions for the following nonlinear integral equation

$$
x(t)=f\left(t, \int_{0}^{\varphi(t)} v\left(t, s, x\left(\gamma_{1}(s)\right)\right) d s, x(\alpha(t))\right) \times
$$




$$
\times g\left(t, x\left(\gamma_{2}(t)\right) \int_{0}^{1} u\left(t, s, x\left(\gamma_{3}(s)\right)\right) d s, x(\beta(t))\right)
$$

for $t \in I=[0,1]$.

In this study, we investigate a more general class of nonlinear integral equations which contain, as particular cases, a lot of integral equations, handled before. Some special cases of Eq.(1.1) have been investigated by various authors. For example, if we take $f(t, y, x)=1$, then Eq.(1.1) can be reduced to the integral equation considered in [11] which arises in models connected with traffic and biology

$$
x(t)=f(t, x(t)) \int_{0}^{1} u(t, s, x(s)) d s .
$$

Similarly, if

$$
f(t, y, x)=1, g(t, y, x)=1+y
$$

and

$$
u(t, s, x)=\frac{t \phi(s) x}{t+s}, \gamma_{2}(t)=\gamma_{3}(t)=t,
$$

then Eq.(1.1) can be reduced to the famous quadratic integral equation of Chandrasekhar type studied in many papers [2,6,10-12] and given of the form

$$
x(t)=1+x(t) \int_{0}^{1} \frac{t}{t+s} \phi(s) x(s) d s .
$$

Finally, Caballero et al. in [9] studied the existence of solutions of following functional-integral equation

$$
\begin{aligned}
x(t)= & f\left(t, \int_{0}^{t} v(t, s, x(s)) d s, x(\alpha(t))\right) \times \\
& \times g\left(t, \int_{0}^{a} x(t) u(t, s, x(s)) d s, x(\beta(t))\right)
\end{aligned}
$$

under the following conditions:

(i) $f, g:[0, a] \times \mathbb{R} \times \mathbb{R} \rightarrow \mathbb{R}$ are continuous and there exists nonnegative constants $c_{i}, d_{i} ;(i=1,2)$ such that

$$
\begin{aligned}
|f(t, 0, x)| & \leq c_{1}+d_{1}|x|, \\
|g(t, 0, x)| & \leq c_{2}+d_{2}|x|,
\end{aligned}
$$

for all $t \in[0, a]$ and $x \in \mathbb{R}$. 
Vol. LII (2014) On the Solutions of a Class of Nonlinear Integral Equations...123

(ii) The functions $f(t, y, x), g(t, y, x)$ satisfy a Lipschitz condition with respect to the variables $y$ and $x$ with constants $k, k^{\prime}$ respectively, i.e.,

$$
\begin{aligned}
\left|f\left(t, y_{1}, x\right)-f\left(t, y_{2}, x\right)\right| & \leq k\left|y_{1}-y_{2}\right| \\
\left|g\left(t, y_{1}, x\right)-g\left(t, y_{2}, x\right)\right| & \leq k\left|y_{1}-y_{2}\right|
\end{aligned}
$$

for all $t \in[0, a]$ and $x, y_{1}, y_{2} \in \mathbb{R}$ and

$$
\begin{aligned}
\left|f\left(t, y, x_{1}\right)-f\left(t, y, x_{2}\right)\right| & \leq k^{\prime}\left|x_{1}-x_{2}\right| \\
\left|g\left(t, y, x_{1}\right)-g\left(t, y, x_{2}\right)\right| & \leq k^{\prime}\left|x_{1}-x_{2}\right|
\end{aligned}
$$

for all $t \in[0, a]$ and $x_{1}, x_{2}, y \in \mathbb{R}$.

(iii) $u, v:[0, a] \times[0, a] \times \mathbb{R} \rightarrow \mathbb{R}$ are continuous.

(iv) $\alpha, \beta:[0, a] \rightarrow[0, a]$ are continuous and satisfy,

$$
\begin{aligned}
\left|\alpha\left(t_{1}\right)-\alpha\left(t_{2}\right)\right| & \leq\left|t_{1}-t_{2}\right|, \\
\left|\beta\left(t_{1}\right)-\beta\left(t_{2}\right)\right| & \leq\left|t_{1}-t_{2}\right|,
\end{aligned}
$$

for all $t_{1}, t_{2} \in[0, a]$.

(v) There exist nonnegative constants $\alpha_{i}, \beta_{i} ;(i=1,2)$ such that

$$
\begin{aligned}
& |v(t, s, x)| \leq \alpha_{1}+\beta_{1}|x|, \\
& |u(t, s, x)| \leq \alpha_{2}+\beta_{2}|x|,
\end{aligned}
$$

for all $t, s \in[0, a]$ and $x \in \mathbb{R}$.

(vi) The inequality

$$
[k(\tilde{\alpha}+\tilde{\beta} r) a+(c+d r)][k(\tilde{\alpha}+\tilde{\beta} r) r a+(c+d r)] \leq r
$$

has a positive solution $r_{0}$, where $\tilde{\alpha}=\max \left\{\alpha_{1}, \alpha_{2}\right\}, \tilde{\beta}=\max \left\{\beta_{1}, \beta_{2}\right\}$, $c=\max \left\{c_{1}, c_{2}\right\}$ and $d=\max \left\{d_{1}, d_{2}\right\}$.

(vii) $k^{\prime}\left[k\left(\tilde{\alpha}+\tilde{\beta} r_{0}\right) a\left(1+r_{0}\right)+2\left(c+d r_{0}\right)\right]<1$.

Theorem 1.1. [9, Theorem 3.1] Equation (1.4) has at least one solution $x \in C[0, a]$ under assumptions $(i)-($ vii $)$. 
It can be verified that if $\varphi(t)=\gamma_{1}(t)=\gamma_{2}(t)=\gamma_{3}(t)=t$, then Eq.(1.1) is reduced to Eq.(1.4) for $a=1$.

Using the technique of a suitable measure of noncompactness in the Banach algebra, we prove an existence theorem for Eq.(1.1). Also, we illustrate our results by suitable examples. The results obtained in this paper generalize several ones obtained up to now. Moreover, our sufficient conditions give the results of [9] under some weaker conditions and for a rather general equation.

\section{Auxiliary facts and notations}

In this section, we give a collection of auxiliary facts which will be needed further on. Assume that $(E,\|\|$.$) is a real Banach space with zero element$ $\theta$. Let $B(x, r)$ denote the closed ball centered at $\mathrm{x}$ and with radius $\mathrm{r}$. The symbol $B_{r}$ stands for the ball $B(\theta, r)$. If $X$ is a subset of $E$, then $\bar{X}$ and $\operatorname{Conv} X$ denote the closure and convex closure of $X$, respectively. With the symbols $\lambda X$ and $X+Y$, we denote the standard algebraic operations on sets. Moreover, we denote by $\mathfrak{M}_{E}$ the family of all nonempty and bounded subsets of $E$ and $\mathfrak{N}_{E}$ its subfamily consisting of all relatively compact subsets. Next we give the concept of a regular measure of noncompactness.

Definition 2.1. [3] A mapping $\mu: \mathfrak{M}_{E} \rightarrow \mathbb{R}^{+}=[0, \infty)$ is said to be a regular measure of noncompactness in $E$ if it satisfies following conditions:

(1) $\mu(X)=0 \Leftrightarrow X \in \mathfrak{N}_{E}$;

(2) $X \subset Y \Rightarrow \mu(X) \leq \mu(Y)$;

(3) $\mu(\bar{X})=\mu(\operatorname{Conv} X)=\mu(X)$;

(4) $\mu(\lambda X)=|\lambda| \mu(X),(\lambda \in \mathbb{R})$;

(5) $\mu(X+Y) \leq \mu(X)+\mu(Y)$;

(6) $\mu(X \cup Y)=\max \{\mu(X), \mu(Y)\}$; 
Vol. LII (2014) On the Solutions of a Class of Nonlinear Integral Equations...125

(7) If $\left\{X_{n}\right\}$ is a sequence of nonempty, bounded, closed subsets of E such that $X_{n+1} \subset X_{n},(n=1,2, \ldots)$ and $\lim _{n \rightarrow \infty} \mu\left(X_{n}\right)=0$, then the set $X_{\infty}=\cap_{n=1}^{\infty} X_{n}$ is nonempty.

In the sequel, we will work in the Banach space $C(I)$ consisting of all real functions defined and continuous on $I=[0,1]$. The space $C(I)$ is furnished with the standard norm

$$
\|x\|=\max \{|x(t)|: t \in I\} .
$$

Obviously, space $C(I)$ also has the structure of the Banach Algebra. We will use a measure of noncompactness in space $C(I)$ which was introduced in [3]. In order to define this measure let us fix a nonempty and bounded subset $X$ of $C(I)$. For $x \in X$ and $\varepsilon \geq 0$ denoted by $w(x, \varepsilon)$ the modulus of continuity of function $\mathrm{x}$, i.e.,

$$
w(x, \varepsilon)=\sup \{|x(s)-x(t)|: t, s \in[0,1],|t-s| \leq \varepsilon\} .
$$

Further let us put

$$
w(X, \varepsilon)=\sup \{w(x, \varepsilon): x \in X\}
$$

and

$$
w_{0}(X)=\lim _{\varepsilon \rightarrow 0} w(X, \varepsilon) .
$$

Function $w_{0}$ is a regular measure of noncompactness in space $C(I)$, [7]. Finally, we recall the fixed point theorem of Darbo. To quote this theorem, we need the following.

Hereafter, we assume unless stated otherwise that $\mu$ is a regular measure of noncompactness in $E$.

Definition 2.2. [3] Let $\Omega$ be a nonempty subset of a Banach space $E$, and let $S: \Omega \rightarrow E$ be a continuous operator that transforms bounded subsets of $\Omega$ onto bounded ones. We will say that $S$ satisfies the Darbo condition (with a constant $k \geq 0$ ) if for any bounded subset $X$ of $\Omega$, we have

$$
\mu(S X) \leq k \mu(X) .
$$

In the case $k<1$, operator $S$ is said to be a contraction (with respect to $\mu$ ).

Theorem 2.3. [7] Let $\Omega$ be a nonempty, bounded, closed and convex subset of space $E$ and let

$$
S: \Omega \rightarrow \Omega
$$

be a continuous transformation such that $\mu(S X) \leq k \mu(X)$ for any nonempty subset $X$ of $\Omega$, where $k \in[0,1)$ is a constant. Then $S$ has a fixed point in set $\Omega$. 
The following theorem is the main tool for our proof.

Theorem 2.4. [8] Assume that $\Omega$ is nonempty, bounded, convex and closed subset of $C(I)$ and operators $F$ and $G$ transform continuously set $\Omega$ into $C(I)$ in such a way that $F(\Omega)$ and $G(\Omega)$ are bounded. Moreover, assume that operator $T=F . G$ transforms $\Omega$ into itself. If operators $F$ and $G$ satisfy Darbo condition on set $\Omega$, with respect to measure of noncompactness $w_{0}$, with constants $k_{1}$ and $k_{2}$, respectively, then operator $T$ satisfies Darbo condition on $\Omega$ with constant

$$
\|F(\Omega)\| k_{2}+\|G(\Omega)\| k_{1}
$$

In particular, if

$$
\|F(\Omega)\| k_{2}+\|G(\Omega)\| k_{1}<1
$$

then $T$ is a contraction with respect to measure of noncompactness $w_{0}$ and so has at least one fixed point in set $\Omega$, where $\|X\|$ is defined by the equality

$$
\|X\|=\sup \{\|x\|: x \in X\}
$$

for any nonempty and bounded subset $X$ of $C(I)$.

\section{The Main Result}

We shall study the existence of the solutions of Eq.(1.1) assuming that following conditions are satisfied:

(i) $f, g: I \times \mathbb{R} \times \mathbb{R} \rightarrow \mathbb{R}$ are continuous. Also, $f(t, y, x)$ and $g(t, y, x)$ satisfy Lipschitz condition with respect to variables $y$ and $x$ with constants $k, k^{\prime}$ respectively, i.e.,

$$
\begin{aligned}
\left|f\left(t, y_{1}, x\right)-f\left(t, y_{2}, x\right)\right| & \leq k\left|y_{1}-y_{2}\right| \\
\left|g\left(t, y_{1}, x\right)-g\left(t, y_{2}, x\right)\right| & \leq k\left|y_{1}-y_{2}\right|
\end{aligned}
$$

for all $t \in I$ and $x, y_{1}, y_{2} \in \mathbb{R}$ and

$$
\begin{aligned}
\left|f\left(t, y, x_{1}\right)-f\left(t, y, x_{2}\right)\right| & \leq k^{\prime}\left|x_{1}-x_{2}\right|, \\
\left|g\left(t, y, x_{1}\right)-g\left(t, y, x_{2}\right)\right| & \leq k^{\prime}\left|x_{1}-x_{2}\right|,
\end{aligned}
$$

for all $t \in I$ and $x_{1}, x_{2}, y \in \mathbb{R}$. 
Vol. LII (2014) On the Solutions of a Class of Nonlinear Integral Equations...127

(ii) $u, v: I \times I \times \mathbb{R} \rightarrow \mathbb{R}$ are continuous and there exist nonnegative constants $\alpha_{i}, \beta_{i}, p_{i} ;(i=1,2)$ such that

$$
\begin{aligned}
& |v(t, s, x)| \leq \alpha_{1}+\beta_{1}|x|^{p_{1}}, \\
& |u(t, s, x)| \leq \alpha_{2}+\beta_{2}|x|^{p_{2}},
\end{aligned}
$$

for all $s, t \in I$ and $x \in \mathbb{R}$.

(iii) $\varphi, \alpha, \beta, \gamma_{j}: I \rightarrow I$ are the continuous functions. $(j=1,2,3)$.

$(i v)$

$$
\left(k \alpha_{1}+m_{1}\right) m_{2}>0,
$$

where $m_{1}$ and $m_{2}$ are the constants such that

$$
|f(t, 0,0)| \leq m_{1} \text { and }|g(t, 0,0)| \leq m_{2}
$$

for all $t \in I$.

$(v)$

$$
\left[k\left(\alpha_{1}+\beta_{1}\right)+m_{1}+k^{\prime}\right]\left[k\left(\alpha_{2}+\beta_{2}\right)+m_{2}+k^{\prime}\right]<1 .
$$

$(v i)$

$$
\begin{aligned}
& k^{\prime}\left[\left(\alpha_{1}+\alpha_{2}+\beta_{1}+\beta_{2}\right)+m_{1}+m_{2}+2 k^{\prime}\right]+ \\
& +k M\left[k\left(\alpha_{1}+\beta_{1}\right)+m_{1}+k^{\prime}\right]<1,
\end{aligned}
$$

where $M$ is the nonnegative constant such that $|u(t, s, x)| \leq M$ for all $t, s \in I$ and $x \in[-1,1]$.

Theorem 3.1. Under assumptions $(i)-(v i)$, there exists at least one $r_{0} \in$ $(0,1)$ such that equation $(1.1)$ has at least one solution $x=x(t)$ belonging to $B_{r_{0}} \subset C(I)$.

Proof. We define continuous function $h:[0,1] \rightarrow \mathbb{R}$ such that

$$
h(r)=\left[k\left(\alpha_{1}+\beta_{1} r^{p_{1}}\right)+m_{1}+k^{\prime} r\right]\left[k r\left(\alpha_{2}+\beta_{2} r^{p_{2}}\right)+m_{2}+k^{\prime} r\right]-r,
$$

where $k, k^{\prime}, m_{i}, \alpha_{i}, \beta_{i}$ and $p_{i}$ for $i \in\{1,2\}$ are the constants given in the assumptions. Then $h(0)>0$ and $h(1)<0$ by assumptions $(i v)$ and $(v)$. 
The continuity of $h$ guarantees that there exists the number $r_{0}$ such that $r_{0} \in(0,1)$ and $h\left(r_{0}\right)=0$.

Now, we shall prove that equation (1.1) has at least one solution $x=x(t)$ belonging to $B_{r_{0}} \subset C(I)$. We define operators $F$ and $G$ on $C(I)$ in the following way:

$$
\begin{aligned}
& (F x)(t)=f\left(t, \int_{0}^{\varphi(t)} v\left(t, s, x\left(\gamma_{1}(s)\right)\right) d s, x(\alpha(t))\right) \\
& (G x)(t)=g\left(t, x\left(\gamma_{2}(t)\right) \int_{0}^{1} u\left(t, s, x\left(\gamma_{3}(s)\right)\right) d s, x(\beta(t))\right) .
\end{aligned}
$$

From the assumptions, $F$ and $G$ transform space $C(I)$ into itself. Further let us define operator $T$ on $C(I)$ by the equality

$$
T x=(F x)(G x) .
$$

Obviously, $T$ transforms $C(I)$ into itself. Since

$$
f(t, 0, x)=f(t, 0, x)-f(t, 0,0)+f(t, 0,0),
$$

we have by $(i)$ that

$$
|f(t, 0, x)| \leq m_{1}+k^{\prime}|x|
$$

for all $t \in I$ and $x \in \mathbb{R}$. Let us fix $x \in C(I)$. Then, using our assumptions for $t \in I$, we get

$$
\begin{aligned}
& |(F x)(t)| \\
= & \left|f\left(t, \int_{0}^{\varphi(t)} v\left(t, s, x\left(\gamma_{1}(s)\right)\right) d s, x(\alpha(t))\right)\right| \\
\leq & \left|f\left(t, \int_{0}^{\varphi(t)} v\left(t, s, x\left(\gamma_{1}(s)\right)\right) d s, x(\alpha(t))\right)-f(t, 0, x(\alpha(t)))\right| \\
& +|f(t, 0, x(\alpha(t)))| \\
\leq & k \int_{0}^{\varphi(t)}\left|v\left(t, s, x\left(\gamma_{1}(s)\right)\right)\right| d s+m_{1}+k^{\prime}|x(\alpha(t))| \\
\leq & k \int_{0}^{\varphi(t)}\left(\alpha_{1}+\beta_{1}\left|x\left(\gamma_{1}(s)\right)\right|^{p_{1}}\right) d s+m_{1}+k^{\prime}|x(\alpha(t))| \\
\leq & k\left(\alpha_{1}+\beta_{1}\|x\|^{p_{1}}\right)+m_{1}+k^{\prime}\|x\| .
\end{aligned}
$$

Similarly, we derive

$$
|(G x)(t)| \leq k\|x\|\left(\alpha_{2}+\beta_{2}\|x\|^{p_{2}}\right)+m_{2}+k^{\prime}\|x\|
$$


Vol. LII (2014) On the Solutions of a Class of Nonlinear Integral Equations...129

for all $x \in C(I)$ and $t \in I$. By (3.1) and (3.2), for $x \in B_{r_{0}}$, we obtain

$$
\begin{aligned}
|(T x)(t)|= & |(F x)(t) \|(G x)(t)| \\
= & {\left[k\left(\alpha_{1}+\beta_{1}\|x\|^{p_{1}}\right)+m_{1}+k^{\prime}\|x\|\right] \times } \\
& {\left[k\|x\|\left(\alpha_{2}+\beta_{2}\|x\|^{p_{2}}\right)+m_{2}+k^{\prime}\|x\|\right] } \\
\leq & {\left[k\left(\alpha_{1}+\beta_{1} r_{0}^{p_{1}}\right)+m_{1}+k^{\prime} r_{0}\right] \times } \\
& {\left[k r_{0}\left(\alpha_{2}+\beta_{2} r_{0}^{p_{2}}\right)+m_{2}+k^{\prime} r_{0}\right] } \\
= & h\left(r_{0}\right)+r_{0} \\
= & r_{0}
\end{aligned}
$$

which implies that $T x \in B_{r_{0}}$.

Now, we shall prove that operator $F$ is continuous on $B_{r_{0}}$. To do this, consider $\varepsilon>0$ and any $x, y \in B_{r_{0}}$ such that $\|x-y\| \leq \varepsilon$. Then,

$$
\begin{aligned}
& |(F x)(t)-(F y)(t)| \\
= & \mid f\left(t, \int_{0}^{\varphi(t)} v\left(t, s, x\left(\gamma_{1}(s)\right)\right) d s, x(\alpha(t))\right)- \\
& -f\left(t, \int_{0}^{\varphi(t)} v\left(t, s, y\left(\gamma_{1}(s)\right)\right) d s, y(\alpha(t))\right) \mid \\
\leq & \mid f\left(t, \int_{0}^{\varphi(t)} v\left(t, s, x\left(\gamma_{1}(s)\right)\right) d s, x(\alpha(t))\right)- \\
& -f\left(t, \int_{0}^{\varphi(t)} v\left(t, s, y\left(\gamma_{1}(s)\right)\right) d s, x(\alpha(t))\right) \mid \\
& +\mid f\left(t, \int_{0}^{\varphi(t)} v\left(t, s, y\left(\gamma_{1}(s)\right)\right) d s, x(\alpha(t))\right)- \\
& -f\left(t, \int_{0}^{\varphi(t)} v\left(t, s, y\left(\gamma_{1}(s)\right)\right) d s, y(\alpha(t))\right) \mid
\end{aligned}
$$

which implies that

$$
\begin{aligned}
& |(F x)(t)-(F y)(t)| \\
\leq & k \int_{0}^{\varphi(t)}\left|v\left(t, s, x\left(\gamma_{1}(s)\right)\right)-v\left(t, s, y\left(\gamma_{1}(s)\right)\right)\right| d s+ \\
& +k^{\prime}|x(\alpha(t))-y(\alpha(t))| \\
\leq & k w_{r_{0}}^{3}(v, \varepsilon)+k^{\prime}\|x-y\| \\
\leq & k w_{r_{0}}^{3}(v, \varepsilon)+k^{\prime} \varepsilon
\end{aligned}
$$


where

$$
w_{r_{0}}^{3}(v, \varepsilon)=\sup \{|v(t, s, x)-v(t, s, y)|: t, s \in I ; x, y \in R ;|x-y| \leq \varepsilon\}
$$

such that $R=\left[-r_{0}, r_{0}\right]$. Notice that, in view of the uniform continuity of function $v$ on set $I \times I \times\left[-r_{0}, r_{0}\right]$, we have that $w_{r_{0}}^{3}(v, \varepsilon) \rightarrow 0$ as $\varepsilon \rightarrow 0$. Thus, above estimate (3.3) shows that operator $F$ is continuous on ball $B_{r_{0}}$. Similarly, we can show that operator $G$ is continuous on ball $B_{r_{0}}$. Obviously, this implies the continuity of operator $T$ on ball $B_{r_{0}}$.

Further, we shall show that operators $F$ and $G$ satisfy Darbo condition on ball $B_{r_{0}}$. In order to do this, let us take a nonempty subset $X$ of ball $B_{r_{0}}$. Fix $\varepsilon>0$ and choose $x \in X$ and $t_{1}, t_{2} \in I$ such that $\left|t_{1}-t_{2}\right| \leq \varepsilon$. Without loss of generality, we may assume that $\varphi\left(t_{2}\right) \leq \varphi\left(t_{1}\right)$. Then, we obtain

$$
\begin{aligned}
& \left|(F x)\left(t_{2}\right)-(F x)\left(t_{1}\right)\right| \\
= & \mid f\left(t_{2}, \int_{0}^{\varphi\left(t_{2}\right)} v\left(t_{2}, s, x\left(\gamma_{1}(s)\right)\right) d s, x\left(\alpha\left(t_{2}\right)\right)\right)- \\
& -f\left(t_{1}, \int_{0}^{\varphi\left(t_{1}\right)} v\left(t_{1}, s, x\left(\gamma_{1}(s)\right)\right) d s, x\left(\alpha\left(t_{1}\right)\right)\right) \mid \\
\leq & \mid f\left(t_{2}, \int_{0}^{\varphi\left(t_{2}\right)} v\left(t_{2}, s, x\left(\gamma_{1}(s)\right)\right) d s, x\left(\alpha\left(t_{2}\right)\right)\right)- \\
& -f\left(t_{2}, \int_{0}^{\varphi\left(t_{1}\right)} v\left(t_{1}, s, x\left(\gamma_{1}(s)\right)\right) d s, x\left(\alpha\left(t_{2}\right)\right)\right) \mid \\
& +\mid f\left(t_{2}, \int_{0}^{\varphi\left(t_{1}\right)} v\left(t_{1}, s, x\left(\gamma_{1}(s)\right)\right) d s, x\left(\alpha\left(t_{2}\right)\right)\right)- \\
& -f\left(t_{1}, \int_{0}^{\varphi\left(t_{1}\right)} v\left(t_{1}, s, x\left(\gamma_{1}(s)\right)\right) d s, x\left(\alpha\left(t_{2}\right)\right)\right) \mid \\
& +\mid f\left(t_{1}, \int_{0}^{\varphi\left(t_{1}\right)} v\left(t_{1}, s, x\left(\gamma_{1}(s)\right)\right) d s, x\left(\alpha\left(t_{2}\right)\right)\right)- \\
& -f\left(t_{1}, \int_{0}^{\varphi\left(t_{1}\right)} v\left(t_{1}, s, x\left(\gamma_{1}(s)\right)\right) d s, x\left(\alpha\left(t_{1}\right)\right)\right) \mid .
\end{aligned}
$$


Vol. LII (2014) On the Solutions of a Class of Nonlinear Integral Equations...131

Hence,

$$
\begin{aligned}
& \left|(F x)\left(t_{2}\right)-(F x)\left(t_{1}\right)\right| \\
\leq & k\left|\int_{0}^{\varphi\left(t_{2}\right)} v\left(t_{2}, s, x\left(\gamma_{1}(s)\right)\right) d s-\int_{0}^{\varphi\left(t_{1}\right)} v\left(t_{1}, s, x\left(\gamma_{1}(s)\right)\right) d s\right| \\
& +w_{r_{0}}^{1}(f, \varepsilon)+k^{\prime}\left|x\left(\alpha\left(t_{2}\right)\right)-x\left(\alpha\left(t_{1}\right)\right)\right| \\
\leq & k \int_{0}^{\varphi\left(t_{2}\right)}\left|v\left(t_{2}, s, x\left(\gamma_{1}(s)\right)\right)-v\left(t_{1}, s, x\left(\gamma_{1}(s)\right)\right)\right| d s+ \\
\quad & +k \int_{\varphi\left(t_{2}\right)}^{\varphi\left(t_{1}\right)}\left|v\left(t_{1}, s, x\left(\gamma_{1}(s)\right)\right)\right| d s+w_{r_{0}}^{1}(f, \varepsilon)+ \\
& +k^{\prime}\left|x\left(\alpha\left(t_{2}\right)\right)-x\left(\alpha\left(t_{1}\right)\right)\right| .
\end{aligned}
$$

Then, by (3.4), we have that

$$
\begin{aligned}
& \left|(F x)\left(t_{2}\right)-(F x)\left(t_{1}\right)\right| \\
\leq & k w_{r_{0}}^{1}(v, \varepsilon)+k L\left|\varphi\left(t_{1}\right)-\varphi\left(t_{2}\right)\right|+w_{r_{0}}^{1}(f, \varepsilon)+ \\
& +k^{\prime}\left|x\left(\alpha\left(t_{2}\right)\right)-x\left(\alpha\left(t_{1}\right)\right)\right| \\
\leq & k w_{r_{0}}^{1}(v, \varepsilon)+k L w(\varphi, \varepsilon)+w_{r_{0}}^{1}(f, \varepsilon)+k^{\prime} w(x, w(\alpha, \varepsilon)),
\end{aligned}
$$

where

$$
\begin{gathered}
w_{r_{0}}^{1}(v, \varepsilon)=\sup \left\{\left|v\left(t_{2}, s, x\right)-v\left(t_{1}, s, x\right)\right|: t_{1}, t_{2}, s \in I, x \in R ;\left|t_{1}-t_{2}\right| \leq \varepsilon\right\}, \\
\left.w_{r_{0}}^{1}(f, \varepsilon)=\sup _{\left.x \in R, y \in[-L, L] ;\left|t_{1}-t_{2}\right| \leq \varepsilon\right\},}, y\right)-f\left(t_{1}, y, x\right) \mid: t_{1}, t_{2} \in I, \\
L=\sup \{|v(t, s, x)|: t, s \in I ; x \in R\}
\end{gathered}
$$

and

$$
w\left(\alpha_{i}, \varepsilon\right)=\sup \left\{\left|\alpha_{i}\left(t_{2}\right)-\alpha_{i}\left(t_{1}\right)\right|: t_{1}, t_{2} \in I ;\left|t_{1}-t_{2}\right| \leq \varepsilon\right\}
$$

for $i=1,2,3$ such that $\alpha_{1}=\varphi, \alpha_{2}=\alpha$ and $\alpha_{3}=x$. From (3.5), we get

$$
w(F x, \varepsilon) \leq k w_{r_{0}}^{1}(v, \varepsilon)+k L w(\varphi, \varepsilon)+w_{r_{0}}^{1}(f, \varepsilon)+k^{\prime} w(x, w(\alpha, \varepsilon)) .
$$

Taking into account the uniform continuity of functions $f, v, \alpha$ and $\varphi$ on the bounded sets, we can deduce by (3.6) that

$$
w_{0}(F X) \leq k^{\prime} w_{0}(X) .
$$


In a similar way, we have

$$
\begin{aligned}
& \left|(G x)\left(t_{2}\right)-(G x)\left(t_{1}\right)\right| \\
= & \mid g\left(t_{2}, x\left(\gamma_{2}\left(t_{2}\right)\right) \int_{0}^{1} u\left(t_{2}, s, x\left(\gamma_{3}(s)\right)\right) d s, x\left(\beta\left(t_{2}\right)\right)\right)- \\
& -g\left(t_{1}, x\left(\gamma_{2}\left(t_{1}\right)\right) \int_{0}^{1} u\left(t_{1}, s, x\left(\gamma_{3}(s)\right)\right) d s, x\left(\beta\left(t_{1}\right)\right)\right) \mid \\
\leq & \mid g\left(t_{2}, x\left(\gamma_{2}\left(t_{2}\right)\right) \int_{0}^{1} u\left(t_{2}, s, x\left(\gamma_{3}(s)\right)\right) d s, x\left(\beta\left(t_{2}\right)\right)\right)- \\
& -g\left(t_{2}, x\left(\gamma_{2}\left(t_{1}\right)\right) \int_{0}^{1} u\left(t_{1}, s, x\left(\gamma_{3}(s)\right)\right) d s, x\left(\beta\left(t_{2}\right)\right)\right) \mid \\
& +\mid g\left(t_{2}, x\left(\gamma_{2}\left(t_{1}\right)\right) \int_{0}^{1} u\left(t_{1}, s, x\left(\gamma_{3}(s)\right)\right) d s, x\left(\beta\left(t_{2}\right)\right)\right)- \\
& -g\left(t_{1}, x\left(\gamma_{2}\left(t_{1}\right)\right) \int_{0}^{1} u\left(t_{1}, s, x\left(\gamma_{3}(s)\right)\right) d s, x\left(\beta\left(t_{2}\right)\right)\right) \mid \\
& +\mid g\left(t_{1}, x\left(\gamma_{2}\left(t_{1}\right)\right) \int_{0}^{1} u\left(t_{1}, s, x\left(\gamma_{3}(s)\right)\right) d s, x\left(\beta\left(t_{2}\right)\right)\right)- \\
& -g\left(t_{1}, x\left(\gamma_{2}\left(t_{1}\right)\right) \int_{0}^{1} u\left(t_{1}, s, x\left(\gamma_{3}(s)\right)\right) d s, x\left(\beta\left(t_{1}\right)\right)\right) \mid \\
\leq & k\left|x\left(\gamma_{2}\left(t_{2}\right)\right) \int_{0}^{1} u\left(t_{2}, s, x\left(\gamma_{3}(s)\right)\right) d s-x\left(\gamma_{2}\left(t_{1}\right)\right) \int_{0}^{1} u\left(t_{1}, s, x\left(\gamma_{3}(s)\right)\right) d s\right| \\
+ & k \mid\left[x \left(w_{r_{0}}^{1}(g, \varepsilon)+k^{\prime}\left|x\left(\beta\left(t_{2}\right)\right)-x\left(\beta\left(t_{1}\right)\right)\right|\right.\right. \\
+ & \left|x\left(\gamma_{2}\left(t_{1}\right)\right) \int_{0}^{1}\left[u\left(t_{2}, s, x\left(\gamma_{3}(s)\right)\right)-u\left(t_{1}, s, x\left(\gamma_{3}(s)\right)\right)\right] d s\right| \\
\hline & \left.\left.\mid \gamma_{2}\left(t_{1}\right)\right)\right] \int_{0}^{1} u\left(t_{2}, s, x\left(\gamma_{3}(s)\right)\right) d s \mid+ \\
\leq & \mid \beta, \varepsilon)) . \\
\leq &
\end{aligned}
$$

By (3.8), we derive that

$$
\begin{aligned}
& \left|(G x)\left(t_{2}\right)-(G x)\left(t_{1}\right)\right| \\
\leq & k M w\left(x, w\left(\gamma_{2}, \varepsilon\right)\right)+k r_{0} w_{r_{0}}^{1}(u, \varepsilon)+w_{r_{0}}^{1}(g, \varepsilon)+k^{\prime} w(x, w(\beta, \varepsilon)),
\end{aligned}
$$

where

$$
\begin{aligned}
w_{r_{0}}^{1}(g, \varepsilon)= & \sup \left\{\left|g\left(t_{2}, y, x\right)-g\left(t_{1}, y, x\right)\right|: t_{1}, t_{2} \in I,\right. \\
& \left.y \in\left[-r_{0} M, r_{0} M\right], x \in R ;\left|t_{1}-t_{2}\right| \leq \varepsilon\right\}, \\
w_{r_{0}}^{1}(u, \varepsilon)= & \sup \left\{\left|u\left(t_{2}, s, x\right)-u\left(t_{1}, s, x\right)\right|: t_{1}, t_{2}, s \in I, x \in R ;\left|t_{1}-t_{2}\right| \leq \varepsilon\right\}
\end{aligned}
$$


Vol. LII (2014) On the Solutions of a Class of Nonlinear Integral Equations...133

and $M$ is the nonnegative constant such that $|u(t, s, x)| \leq M$ for all $t, s \in I$ and $x \in[-1,1]$. Also,

$$
w\left(\beta_{j}, \varepsilon\right)=\sup \left\{\left|\beta_{j}\left(t_{2}\right)-\beta_{j}\left(t_{1}\right)\right|: t_{1}, t_{2} \in I ;\left|t_{1}-t_{2}\right| \leq \varepsilon\right\}
$$

for $j=1,2,3$ such that $\beta_{1}=\beta, \beta_{2}=\gamma_{2}$ and $\beta_{3}=x$. From (3.9), we get

$$
\begin{aligned}
w(G x, \varepsilon) \leq & k M w\left(x, w\left(\gamma_{2}, \varepsilon\right)\right)+k r_{0} w_{r_{0}}^{1}(u, \varepsilon)+w_{r_{0}}^{1}(g, \varepsilon)+ \\
& +k^{\prime} w(x, w(\beta, \varepsilon)) .
\end{aligned}
$$

Since functions $\gamma_{2}, u, g$ and $\beta$ are uniform continuous on the bounded sets, we can obtain by (3.10) that

$$
w_{0}(G X) \leq\left(k^{\prime}+k M\right) w_{0}(X) .
$$

Finally, linking (3.1), (3.2), (3.7), (3.11) and Theorem 2.4, we get that $T$ satisfies Darbo condition on ball $B_{r_{0}}$ with constant $\tilde{k}$ such that

$$
\begin{aligned}
\tilde{k}= & k^{\prime}\left[k\left(\alpha_{1}+\beta_{1} r_{0}^{p_{1}}\right)+m_{1}+k^{\prime} r_{0}+k r_{0}\left(\alpha_{2}+\beta_{2} r_{0}^{p_{2}}\right)+m_{2}+k^{\prime} r_{0}\right]+ \\
& +k M\left[k\left(\alpha_{1}+\beta_{1} r_{0}^{p_{1}}\right)+m_{1}+k^{\prime} r_{0}\right] .
\end{aligned}
$$

Since the inequality

$k^{\prime}\left[k\left(\alpha_{1}+\alpha_{2}+\beta_{1}+\beta_{2}\right)+m_{1}+m_{2}+2 k^{\prime}\right]+k M\left[k\left(\alpha_{1}+\beta_{1}\right)+m_{1}+k^{\prime}\right]<1$

holds by assumption (vi), $\tilde{k}<1$ and so $T$ is a contraction on ball $B_{r_{0}}$ and has a fixed point in $B_{r_{0}}$ by Theorem 2.4. Consequently, Eq.(1.1) has at least one solution in $B_{r_{0}}$. This step completes the proof of our theorem.

Note 3.1. Functions $u, v, \alpha$ and $\beta$ satisfying conditions (iii), (iv) and $(v)$ of Theorem 3.1 in [9] for $a=1$ also satisfy conditions (ii) and (iii) of our theorem. But, converse of this may not be correct.

\section{Examples}

In this section, we present some examples verifying the conditions of Theorem 3.1 and not verifying the conditions of Theorem 3.1 in [9].

Example 4.1. Let us take functions $f, g: I \times \mathbb{R} \times \mathbb{R} \rightarrow \mathbb{R}$ defined by

$$
f(t, y, x)=\frac{[\exp (t-1)+1] \sin x}{5+(t-1)^{2}}+\frac{y}{5+t^{2}}
$$


and

$$
g(t, y, x)=\frac{2 \sin \left(|x|+t^{2}\right)}{5+t^{3}}+\frac{y}{5+t^{2}} .
$$

These functions are continuous and satisfy Lipschitz condition with respect to variables $y$ and $x$ with constants $k=1 / 5$ and $k^{\prime}=2 / 5$, respectively. Also, since

$$
f(t, 0,0)=0 \text { and } g(t, 0,0)=\frac{2 \sin \left(t^{2}\right)}{5+t^{3}},
$$

we can choose nonnegative constants $m_{1}$ and $m_{2}$ as $m_{1}=0$ and $m_{2}=2 / 5$. Next, we take

$$
v(t, s, x)=\frac{\left[1+\cos \left(t s^{3}\right)\right]\left(x^{2}+s\right)}{3+s^{2}}
$$

and

$$
u(t, s, x)=\frac{\sin \sqrt{t}+\sqrt{|x|^{3}}}{5+\ln (1+s)+s^{3}} .
$$

It is easy to verify that the inequalities

$$
|v(t, s, x)| \leq \frac{2}{3}+\frac{2}{3}|x|^{2} \text { and }|u(t, s, x)| \leq \frac{1}{5}+\frac{1}{5}|x|^{\frac{3}{2}}
$$

hold for all $t, s \in I=[0,1]$ and $x \in \mathbb{R}$. So, assumption (ii) is satisfied with

$$
\alpha_{1}=\beta_{1}=\frac{2}{3}, \alpha_{2}=\beta_{2}=\frac{1}{5}, p_{1}=2 \text { and } p_{2}=\frac{3}{2} .
$$

On the other hand, if we take

$$
\alpha(t)=\beta(t)=\sqrt{t}, \varphi(t)=t^{2} \text { and } \gamma_{1}(t)=\gamma_{2}(t)=\gamma_{3}(t)=\frac{t}{2},
$$

assumption (iii) holds.

Furthermore, since

$$
\begin{gathered}
\left(k \alpha_{1}+m_{1}\right) m_{2}>0 \\
{\left[k\left(\alpha_{1}+\beta_{1}\right)+m_{1}+k^{\prime}\right]\left[k\left(\alpha_{2}+\beta_{2}\right)+m_{2}+k^{\prime}\right]=\frac{44}{75}<1}
\end{gathered}
$$

and

$$
\begin{aligned}
& k^{\prime}\left[k\left(\alpha_{1}+\alpha_{2}+\beta_{1}+\beta_{2}\right)+m_{1}+m_{2}+2 k^{\prime}\right]+ \\
& +k M\left[k\left(\alpha_{1}+\beta_{1}\right)+m_{1}+k^{\prime}\right]=\frac{252}{375}<1,
\end{aligned}
$$

the inequalities in assumptions $(i v),(v)$ and $(v i)$ hold, where

$$
M=\sup \{|u(t, s, x)|: t, s \in I ; x \in[-1,1]\}=\frac{2}{5} .
$$


Vol. LII (2014) On the Solutions of a Class of Nonlinear Integral Equations...135

Then, Eq.(1.1) has the form

$$
\begin{aligned}
x(t)= & \left(\frac{[\exp (t-1)+1] \sin x(\sqrt{t})}{5+(t-1)^{2}}+\right. \\
& \left.+\frac{1}{5+t^{2}} \int_{0}^{t^{2}} \frac{\left[1+\cos \left(t s^{3}\right)\right]\left[\left(x\left(\frac{s}{2}\right)\right)^{2}+s\right]}{3+s^{2}} d s\right) \times \\
& \left(\frac{2 \sin \left(|x(\sqrt{t})|+t^{2}\right)}{5+t^{3}}+\frac{x\left(\frac{t}{2}\right)}{5+t^{2}} \int_{0}^{1} \frac{\sin \sqrt{t}+\sqrt{\left|x\left(\frac{s}{2}\right)\right|^{3}}}{5+\ln (1+s)+s^{3}} d s\right)
\end{aligned}
$$

where $t \in[0,1]$. Consequently, by applying Theorem 3.1, we deduce that Eq.(4.1) has at least one solution $x \in C[0,1]$.

Since there is no constants $\alpha_{1}, \beta_{1}, \alpha_{2}$ and $\beta_{2}$ satisfying the inequalities

$$
|v(t, s, x)| \leq \alpha_{1}+\beta_{1}|x| \text { and }|u(t, s, x)| \leq \alpha_{2}+\beta_{2}|x|
$$

for all $t, s \in I=[0,1]$ and $x \in \mathbb{R}$, if we take

$$
\gamma_{1}(t)=\gamma_{2}(t)=\gamma_{3}(t)=\varphi(t)=t, \alpha(t)=\beta(t)=\sqrt{t},
$$

the result in [9] is inapplicable to the integral equation

$$
\begin{aligned}
x(t)= & \left(\frac{[\exp (t-1)+1] \sin x(\sqrt{t})}{5+(t-1)^{2}}+\right. \\
& \left.+\frac{1}{5+t^{2}} \int_{0}^{t} \frac{\left[1+\cos \left(t s^{3}\right)\right]\left[(x(s))^{2}+s\right]}{3+s^{2}} d s\right) \times \\
& \times\left(\frac{2 \sin \left(|x(\sqrt{t})|+t^{2}\right)}{5+t^{3}}+\frac{x(t)}{5+t^{2}} \int_{0}^{1} \frac{\sin \sqrt{t}+\sqrt{|x(s)|^{3}}}{5+\ln (1+s)+s^{3}} d s\right) .
\end{aligned}
$$

Also, functions $\alpha$ and $\beta$ don't hold the inequalities in condition (iv) of Theorem 3.1 in [9].

Example 4.2. If we take

$f(t, y, x)=a(t)+y, g(t, y, x)=1, \varphi(t)=\gamma_{1}(t)=\alpha(t)=t$ and $u(t, s, x)=0$

for all $t, s \in I ; x, y \in \mathbb{R}$, then Eq.(1.1) is reduced to well known nonlinear Volterra integral equation

$$
x(t)=a(t)+\int_{0}^{t} v(t, s, x(s)) d s,
$$


where function $a: I \rightarrow \mathbb{R}$ is continuous. Also, we assume that there exist nonnegative constants $\alpha_{1}, \beta_{1}$ and $p_{1}$ such that the inequality

$$
|v(t, s, x)| \leq \alpha_{1}+\beta_{1}|x|^{p_{1}}
$$

holds for all $t, s \in I$ and $x \in \mathbb{R}$.

The theory of above equation (4.2) is well developed in [1,4,5,13,14]. For this specific choice of $f, g, \varphi, \gamma_{1}, \alpha$ and $u$, assumptions ( $\left.i\right)$, (ii) and (vi) are satisfied with

$$
k=1, k^{\prime}=0, \alpha_{1}, \beta_{1}, \alpha_{2}=\beta_{2}=0, m_{1}=\|a\|, m_{2}=1 \text { and } M=0 .
$$

Assumption (iii) already holds for $\varphi, \gamma_{1}, \alpha$ and any continuous functions $\beta, \gamma_{2}, \gamma_{3}: I \rightarrow I$.

Additionally, we assume that the inequalities in conditions $(i v)$ and $(v)$ which are equivalent to

$$
\alpha_{1}+\|a\|>0
$$

and

$$
\alpha_{1}+\beta_{1}+\|a\|<1,
$$

respectively are verified.

Then, we deduce from Theorem 3.1 that there exists at least one number $r_{0} \in(0,1)$ and Eq.(4.2) has at least one solution $x=x(t)$ belonging to $B_{r_{0}} \subset C[0,1]$.

We put

$$
v(t, s, x)=\frac{15 t^{2}+4 \sin \left(\frac{x}{1+x^{2}}\right)}{19+\exp (1-t)}, a(t)=\frac{1}{t^{2}+25} .
$$

The inequality

$$
\begin{aligned}
|v(t, s, x)| & \leq \frac{15}{20}+\frac{4}{20}\left|\sin \left(\frac{x}{1+x^{2}}\right)\right| \\
& \leq \frac{3}{4}+\frac{1}{5}\left|\frac{x}{1+x^{2}}\right| \\
& \leq \frac{3}{4}+\frac{1}{5}|x|
\end{aligned}
$$

holds for all $t, s \in I$ and $x \in \mathbb{R}$.

So (4.3), (4.4) and (4.5) are satisfied with

$$
\alpha_{1}=\frac{3}{4}, \beta_{1}=\frac{1}{5}, p_{1}=1 \text { and }\|a\|=\frac{1}{25} .
$$


Vol. LII (2014) On the Solutions of a Class of Nonlinear Integral Equations...137

Then, our integral equation takes the form

$$
x(t)=\frac{1}{t^{2}+25}+\int_{0}^{t} \frac{15 t^{2}+4 \sin \left(\frac{x(s)}{1+x^{2}(s)}\right)}{19+\exp (1-t)} d s
$$

which has at least one solution $x \in B_{r_{0}} \subset C[0,1]$.

On the other hand, the inequality given in assumption (vi) of Theorem 3.1 in [9] doesn't hold with

$$
k=1, \tilde{\alpha}=\frac{3}{4}, \tilde{\beta}=\frac{1}{5}, a=1, c=1 \text { and } d=0,
$$

since

$$
[k(\tilde{\alpha}+\tilde{\beta} r) a+(c+d r)][k(\tilde{\alpha}+\tilde{\beta} r) r a+(c+d r)]>r
$$

for all $r \in(0, \infty)$. Hence, the result in $[9]$ is inapplicable to integral equation (4.6).

Example 4.3. Let us define

$f(t, y, x)=1, g(t, y, x)=a(t)+y, \gamma_{2}(t)=\gamma_{3}(t)=\beta(t)=t$ and $v(t, s, x)=0$

for all $t, s \in I$ and $x, y \in \mathbb{R}$, where function $a: I \rightarrow \mathbb{R}$ is continuous.

It is known from [9] that function u given as

$$
u(t, s, x)= \begin{cases}0, & s=0, t \geq 0, x \in \mathbb{R} \\ \frac{t}{t+s} \phi(s) x, & s \neq 0, t \geq 0, x \in \mathbb{R}\end{cases}
$$

is continuous on $I \times I \times \mathbb{R}$. Here, $\phi: I \rightarrow \mathbb{R}$ is continuous and $\phi(0)=0$. For these functions, Eq.(1.1) has the form

$$
x(t)=a(t)+x(t) \int_{0}^{1} \frac{t}{t+s} \phi(s) x(s) d s
$$

which is related with the Chandrasekhar equation considered in [2,6, 10-12]. In this example, $f$ and $g$ satisfy Lipschitz condition with respect to variables $y$ and $x$ with constants $k=1$ and $k^{\prime}=0$, respectively. Also, since

$$
f(t, 0,0)=1, g(t, 0,0)=a(t), v(t, s, x)=0 \text { and }|u(t, s, x)| \leq\|\phi\||x|
$$

for all $t, s \in I$ and $x \in \mathbb{R}$, we can choose nonnegative constants $m_{1}, m_{2}, \alpha_{1}$, $\beta_{1}, \alpha_{2}, \beta_{2}$ and $M$ as

$$
m_{1}=1, m_{2}=\|a\|, \alpha_{1}=\beta_{1}=\alpha_{2}=0, \beta_{2}=\|\phi\| \text { and } M=\|\phi\| .
$$


Therefore, conditions $(i)$ and $(i i)$ are satisfied. It is obvious that condition (iii) is satisfied for $\beta, \gamma_{2}, \gamma_{3}$ and any continuous functions $\varphi, \gamma_{1}, \alpha: I \rightarrow I$. The inequalities in conditions (iv), $(v)$ and $(v i)$ can be expressed as

$$
\begin{gathered}
\|a\|>0, \\
\|a\|+\|\phi\|<1
\end{gathered}
$$

and

$$
\|\phi\|<1
$$

respectively. If (4.8) and (4.9) which implies (4.10) hold, we have by Theorem 3.1 that there exists at least one number $r_{0} \in(0,1)$ and Eq.(4.7) has at least one solution $x=x(t)$ belonging to $B_{r_{0}} \subset C[0,1]$.

If we take

$$
a(t)=\frac{e^{t-1}}{\sin ^{2}(t-1)+25}, \phi(t)=\frac{7 t}{10},
$$

then (4.8) and (4.9) hold which imply that the equation

$$
x(t)=\frac{e^{t-1}}{\sin ^{2}(t-1)+25}+\frac{7 t}{10} x(t) \int_{0}^{1} \frac{s}{t+s} x(s) d s
$$

has at least one solution $x \in C[0,1]$.

But, the inequality given in assumption (vi) of Theorem 3.1 in [9] doesn't hold for constants

$$
k=1, \tilde{\alpha}=0, \tilde{\beta}=\frac{7}{10}, a=1, c=1 \text { and } d=0,
$$

since

$$
[k(\tilde{\alpha}+\tilde{\beta} r) a+(c+d r)][k(\tilde{\alpha}+\tilde{\beta} r) r a+(c+d r)]>r
$$

for all $r \in(0, \infty)$. So, the result in [9] is inapplicable to integral equation (4.11).

Example 4.4. Let us consider the following nonlinear integral equation of the form

$$
\begin{aligned}
x(t)= & \frac{\ln (t+e-1) \sin x\left(t^{3}\right)}{t+19}+ \\
& +\frac{1}{t+4} \int_{0}^{t}\left(\frac{4 s \sin x(s)}{20 s^{2}+5}+\frac{10 \ln (s+e-1)}{s+3}\right) d s .
\end{aligned}
$$


Vol. LII (2014) On the Solutions of a Class of Nonlinear Integral Equations...139

Put

$$
\begin{gathered}
f(t, y, x)=\frac{\ln (t+e-1) \sin x}{t+19}+\frac{y}{t+4}, g(t, y, x)=1 \\
v(t, s, x)=\frac{4 s \sin x}{20 s^{2}+5}+\frac{10 \ln (s+e-1)}{s+3}, u(t, s, x)=0 \\
\gamma_{1}(t)=\varphi(t)=t, \alpha(t)=t^{3}
\end{gathered}
$$

and

$$
k=\frac{1}{4}, k^{\prime}=\frac{1}{20}, m_{1}=0, m_{2}=1, \alpha_{1}=\frac{5}{2}, \beta_{1}=\frac{1}{5}, \alpha_{2}=0, \beta_{2}=0, M=0 .
$$

It is easy to show that all of the conditions of Theorem 3.1 hold. Therefore, Theorem 3.1 guarantees that there exists at least one $r_{0} \in(0,1)$ such that Eq.(4.12) has at least one solution $x=x(t)$ belonging to $B_{r_{0}} \subset C[0,1]$.

On the other hand, the inequality given in assumption (vi) of Theorem 3.1 in [9] doesn't hold for constants

$$
\begin{gathered}
k=\frac{1}{4}, \alpha_{1}=\frac{5}{2}, \alpha_{2}=0, \beta_{1}=\frac{1}{5}, \beta_{2}=0, a=1, \\
c_{1}=0, c_{2}=1, d_{1}=\frac{1}{20} \text { and } d_{2}=0 .
\end{gathered}
$$

Hence, the result in [9] is inapplicable to integral equation (4.12).

\section{Acknowledgements}

We would like to thank the referee for the help and suggestions which have improved the paper.

\section{References}

[1] R. P. Agarval, D. O'Regan, and Wong P. J. Y., Positive solutions of differential, difference and integral equations, Kluwer Academic, Dordrecht, 1999.

[2] I. K. Argyros, Quadratic equations and applications to Chandrasekhar's and related equations, Bull. Austral. Math. Soc., 32, (1985), 275-292.

[3] J. Banaś and K. Goebel, Measures of noncompactness in Banach space, Lecture Notes in Pure and Applied Mathematics, New York: Dekker, 60, 1980.

[4] J. Banaś, Integrable solutions of Hammerstein and Urysohn integral equations, $J$. Austral. Math. Soc., 46, (1989), 61-68. 
[5] J. Banaś, Applications of measure of weak compactness and some classes of operators in the theory of functional equations in the Lebesque space, Nonlinear Anal., 30, (1997), 3283-3293.

[6] J. Banaś, M. Lecko, and W. G. El-Sayed, Existence theorems for some quadratic integral equations, J. Math. Anal. Appl., 222, (1998), 276-285.

[7] J. Banaś and M. Lecko, Fixed points of the product of operators in Banach algebra, Panamer. Math. J., 12, (2002), 101-109.

[8] J. Banaś and L. Olszowy, On a class of mesures of noncompactness in Banach algebras and their application to nonlinear integral equations, J. for Analy. and its Appl., 28, (2009), 1-24.

[9] J. Caballero, A. B. Mingarelli, and K. Sadarangani, Existence of solutions of an integral equation of Chandrasekhar type in the theory of radiative transfer, Electron. J. Differential Equations, 57, (2006), 1-11.

[10] S. Chandrasekhar, Radiative Transfer, Oxford University Press, London, 1950.

[11] K. Deimling, Nonlinear Functional Analysis, Springer- Verlag, Berlin, 1985.

[12] S. Hu, M. Khavanin, and W. Zhuang, Integral equations arising in the kinetic theory of gases, Appl. Analysis, 34, (1989), 261-266.

[13] A. Karoui, On the existence of continuous solutions of nonlinear integral equations, Appl. Math. Lett., 18, (2005), 299-305.

[14] P. P. Zabrejko, A. I. Koshelev, M. A. Krasnosel'skii, S. G. Mikhlin, Rakovshchik L. S., and Stecenko V. J., Integral equations, Noordhoff, Leyden, 1975 .

İsmet Özdemir

İnönü Üniversitesi, Eğitim Fakültesi, A-Blok,

Malatya, 44280, Turkey

E-mail: ismet.ozdemir@inonu.edu.tr

Bekir İlhan

Malatya Fen Lisesi, Malatya, 44110, Turkey

E-mail: bekirilhan@gmail.com

Ümit Çakan

Nevşehir Hacı Bektaş Veli Üniversitesi, Fen Edebiyat Fakültesi,

Matematik Bölümü, Nevşehir, 50300, Turkey

E-mail: umitcakan@nevsehir.edu.tr

Received: 30.01.2014

Accepted: 12.05.2014 